\title{
FABRICATION OF AN ACTIVE NANOSTENCIL WITH INTEGRATED MICROSHUTTERS
}

\author{
R.W. Tjerkstra $^{(1)}$, P. Ekkels ${ }^{(1)}$, G. Krijnen ${ }^{(1)}$, S. Egger ${ }^{(2)}$, E. Berenschot ${ }^{(1)}$, K.C. Ma ${ }^{(1)}$ and J. Brugger ${ }^{(3)}$ \\ ${ }^{(1)} \mathrm{MESA}^{+}$research institute, ${ }^{(2)}$ University of Cambridge, ${ }^{(3)}$ Ecole polytechnique federale de Lausanne \\ G.J.M.Krijnen@utwente.nl
}

\begin{abstract}
An active nanostencil, consisting of a thin $(200 \mathrm{~nm})$ silicon nitride membrane with attached polysilicon microactuators that can be used to dynamically open and/or close holes in the silicon nitride membrane, is presented. This nanostencil can be used as a shadow mask in an evaporation setup. Main features of the nanostencil are the absence of sacrificial oxide in the final product, strengthening of the membrane by a polysilicon hexagonal structure that is attached directly to the membrane and the use of low-doped regions in the polysilicon to separate the stator and rotor electrically.
\end{abstract}

\section{INTRODUCTION}

One of the main advantages of evaporating through a shadow mask is the fact that the surface of the sample stays clean. Therefore also vulnerable samples that would not survive the lithography process can be patterned.

During evaporation through a nanostencil, patterns can be made by moving the sample with regard to the stencil. By changing the speed of the sample, the thickness of the deposited wires can be varied locally. In this way patterns with interesting quantum properties (like electron localization) can be made. The successful evaporation of gold dots through a nanostencil has been demonstrated [1 - 4]. With the addition of moveable shutters which open, close, or change the shape or size of openings in the nanostencil many new possibilities occur, depending on the shapes of the actuators and the openings in the nanostencil. The fabrication of Single Electron Transistors and other quantum devices will be greatly facilitated when shutters are incorporated on the nanostencil. We call such a nanostencil an 'active nanostencil', as opposed to a 'passive nanostencil', which doesn't contain moveable structures. In this paper the design, fabrication and characterization of an active nanostencil is presented.

\section{DESIGN OF THE NANOSTENCLL}

The active nanostencil consists of a thin membrane containing openings, on which the shutter structures are fabricated using micromachining techniques. To achieve a high resolution during evaporation the sample has to be very close to the nanostencil (a distance in the order of $100 \mathrm{~nm}$ is preferred). Because the shutters have to be several micrometers thick in order to have enough stiffness in the $z$-direction the sample has to approach the membrane below (the side where the silicon is) as shown in figure 1 (the stencil is mounted upside-down in the evaporator). However, this calls for rather large membranes in order to allow for reasonably sized, and thus easy to manipulate samples. As can be seen' in figure 1, the materials that are evaporated are also deposited on the actuators.

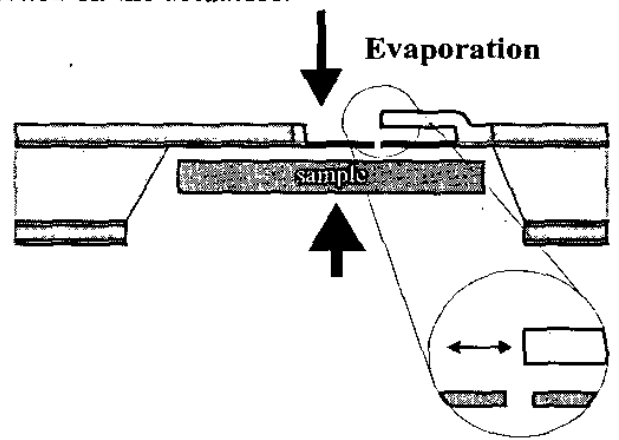

Figure 1: arrangement of the nanostencil and sample

\section{The membrane}

Membrane sizes of $4 \times 4 \mathrm{~mm}^{2}$ on which functional structures were attached directly were fabricated. The membrane thickness was chosen to be only $100-200$ $\mathrm{nm}$. The openings' in a thicker membrane will clog faster during evaporation, but a thinner membrane will be more fragile. Etching holes in a thicker membrane using a Focussed Ion Beam etcher (FIB) is difficult because $\mathrm{SiN}$ is a non-conducting material and not very suitable for FlB etching.

\section{The supporting structure}

The thin membrane is very vulnerable for process- and handling-induced stress. Therefore a supporting structure was made on top of the membrane. This prevents the membrane from deforming and/or breaking under heavy load. The support consists of a hexagonal pattern made in polysilicon, to which the microactuators are connected. The three most important purposes for which the supporting structure is used are:

- give support to the membrane

- provide electrical connections to the actuators

- provide physical connections for theactuators.

The material that is best suited for these purposes is polysilicon [7]. This material is easily applicable, has the right physical properties and, when doped with boron, conducts electricity well enough to provide electrical connections to the actuators. It also can be made reasonably stress-free, which is important for the fabrication of flat membranes. For the supporting structure a hexagonal framework with side beams of $170 \mu \mathrm{m}$ in length and $50 \mu \mathrm{m}$ in width was chosen. This shape is relatively stiff, can be used to apply a voltage to all actuators and has large enough openings to contain the actuators. The supporting polysilicon 
hexagonal structure is attached directly to the silicon nitride membrane.

\section{Design of the actuator}

Considering the difficulties of stress and temperature control in UHV thermal actuation of the shutters on the membrane was abandoned. Due to its relative simplicity over electromagnetic and piezoelectric actuation and its favorable scaling properties [5] electrostatic actuation was eventually chosen.
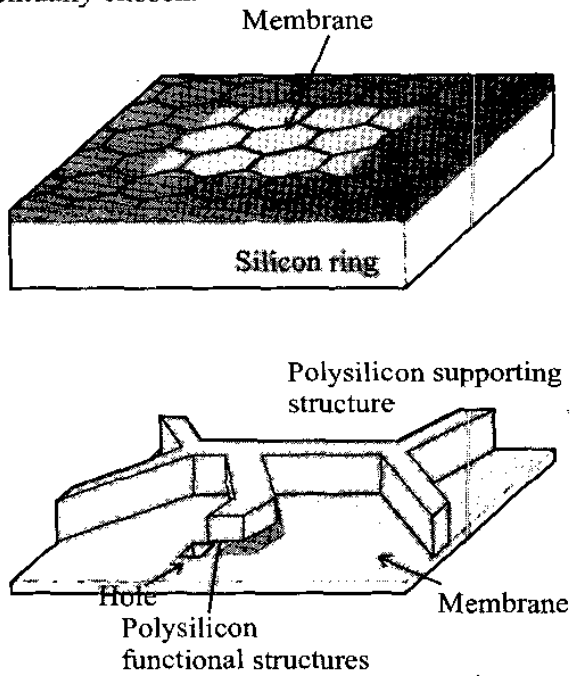

Figure 2: schematic view of the nanostencil. The lower part of the picture shows a small part of the membrane

The actuators are made in polysilicon and directly integrated in the design of the supporting structure (figure 2). They are made free-standing using a sacrificial oxide layer that is positioned beneath the actuators but not beneath the supporting structure (see section 4). To demonstrate the applicability and functionality of our approach a design of a simple electrostatic comb-drive structure, fitting into a hexagonal unit-cell, has been implemented. An actuator-spring 'construction with the following properties is needed:

- The construction has to fit into one hexagonal unit cell of the pattern (length of one side 170 $\mu \mathrm{m})$.

- The movement of the actuator should be at least $1 \mu \mathrm{m}$.

- The actuator should not be anchored on the underlying membrane to prevent bending of the membrane

- The required voltage should preferably be low to be able to use it practically in an UHV setup

- The actuator must be able to stand accelerations in the $x-y-z$ directions due to normal handling without getting damaged or out of order. The stiffness in $\mathrm{y}$ and $\mathrm{z}$ directions has to be sufficient in order to withstand the extra stress caused by the evaporation of material on the actuator.

The structure is anchored to the hexagonal framework on two points that are electrically isolated from each other. The dimensions of the actuator/spring design are the result of an optimisation of the actuators for the available surface area. The vertical stiffness of the structure is high due to the thickness of the polysilicon layer $(5 \mu \mathrm{m})$, thus minimising the chance of the actuator getting into contact with the membrane.

The displacement of the comb-fingers as a function of the comb-drive parameters and the spring-stiffness $k_{s}$ is given by:

$$
x=\frac{n_{c} \varepsilon_{0} h}{k_{s} \cdot g} U^{2}
$$

with nc the number of finger pairs, in this case $29, \mathrm{~d}$ the distance between the fingers of the comb, $h$ the height of the comb-fingers and $\varepsilon_{0}$ the permittivity of vacuum. To make optimal use of the area in a cell a double spring is folded 14 times to get a stiffness $k_{s}$ of:

$$
k_{s}=\frac{1}{7} E_{S i} \frac{h \omega^{3}}{L^{3}}
$$

with $\mathrm{L}$ the length of a single spring, $\mathrm{h}$ the thickness of the spring, $w$ the width of the spring and $E_{S i}$ the Youngs modulus of polysilicon. Using $2 \mu \mathrm{m}$ wide polysilicon beams and $2 \mu \mathrm{m}$ wide gaps the micro-actuator is designed to have a displacement of $1.6 \mu \mathrm{m}$ at a voltage of 20 Volt. Figure 4 shows the final design of the hexagonal support structure containing the comb drive actuator

\section{Local doping}

To connect the actuator to the necessary electrical potentials to operate it the supporting structure is doped with boron. Initially an undoped polysilicon support layer is deposited. The layer is doped afterwards with boron by diffusion. To separate the rotor from the stator and thus prevent short-circuiting an $\mathrm{SiO}_{2}$ mask is used during boron doping to locally prevent diffusion of boron into the polysilicon. Electrical isolation between the individual contacts is obtained by the relatively low conduction of the undoped polysilicon.

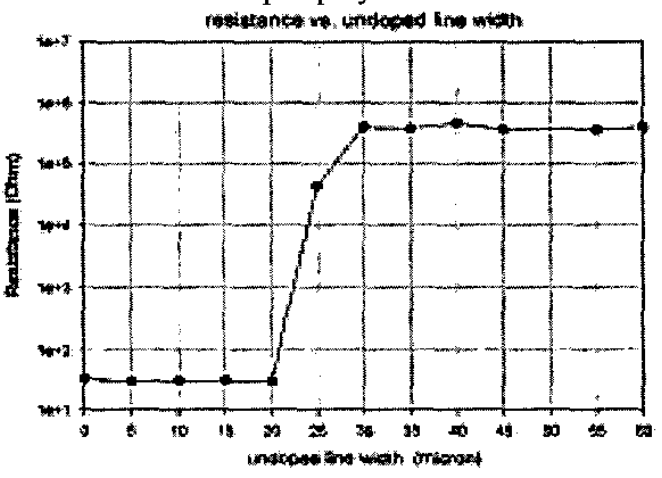

Figure 3: resistance of a $4 \mathrm{~mm}$ wide intrinsic polysilicon beam as a function of the length of the beam

To determine the required dimensions for isolation a polysilicon line of $4 \mu \mathrm{m}$ wide was investigated for various lengths of the $\mathrm{SiO}_{2}$ mask used during doping. The results showed that a distance of $30 \mu \mathrm{m}$ between two regions that were unmasked during doping is sufficient to create a barrier with a high enough electrical resistance to effectively isolate two individual electrical areas (approx.' $700 \mathrm{k} \Omega$, see figure 3). Using 
White Light Interference Microscopy (WLIM) it was experimentally verified that local doping gave only rise to small $(20-50 \mathrm{~nm})$ deformations of the membrane.

\section{REALISATION AND TESTING}

\section{Cleanroom processing}

The eventual fabrication process for the active nanostencils is as follows (figure 4). In order to get flat SiN membranes a $200 \mathrm{~nm}$ SiN layer is deposited directly onto a bare $<100>4$ " SSP Si wafer (A). Next the $\mathrm{SiN}$ layer is covered with a $\mathrm{SiO}_{2}$ layer of $1.5 \mu \mathrm{m}$ by TEOS deposition, and subsequently patterned (B). After patterning, the $\mathrm{SiO}_{2}$ is only present at positions where free-standing parts of the actuators are required. Next a poly-silicon layer of $5 \mu \mathrm{m}$ is deposited (C), doped locally with boron by Solid Source Dotation (D) (using a $\mathrm{SiO}_{2}$ mask not shown in figure 4), and patterned by RIE-etching (E). In the next steps the membrane is formed by $\mathrm{KOH}$ etching from the back side of the wafer (F). In order to protect the poly-silicon during $\mathrm{KOH}$ etching the structures are covered with a $200 \mathrm{~nm} \mathrm{SiN}$ protection layer which is removed later by RIE-etching after the membranes are formed by etching from the backside. Before this layer is deposited the $\mathrm{SiO}_{2}$ is etched isotropically in BHF to create an underetch of $200 \mathrm{~nm}$, which is later filled with $\mathrm{SiN}$ to yield so-called 'anti stiction feet' [6]. This leaves the actuators with a thin SiN layer on the sides which acts as protection against short-circuiting in the case the fingers of the rotor and stator touch. Finally the actuators are etched free in a BHF solution $(G)$ during sacrificial layer etching in which all silicon oxide is removed. After this step the actuators are rinsed thoroughly with water and IPA, and subsequently immersed in cyclohexane. Then they are released using freeze-drying [7]. On one 4" wafer about 50 active nanostencils could be made.

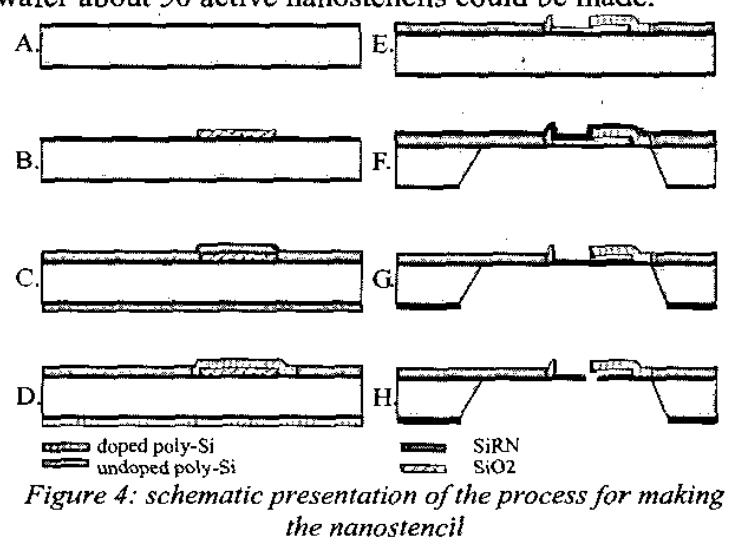

\section{Results}

After the membranes are formed (F) they are highly deformed. Due to the compressive stress of the $\mathrm{SiO}_{2}$ sacrificial layer, deformations of up to $100 \mu \mathrm{m}$ have been measured. After the sacrificial etch of $\mathrm{SiO}_{2}$ the membranes return to an almost perfect flat state. The curvature of the membranes was measured using White Light Interference Microscopy. The overall curvature over a quarter of the membrane was $50 \mathrm{~nm}$ with steps of $100 \mathrm{~nm}$ of the SiN between the hexagonal patchwork.

The hexagonal supporting structure turns out to be a good way to prevent damaging of entire membranes. Imperfections in the framework were seen to be limited at most to neighboring cells. In figure 5 a polysilicon actuator that is attached to a silicon nitride membrane via a hexagonal supporting structure is shown.

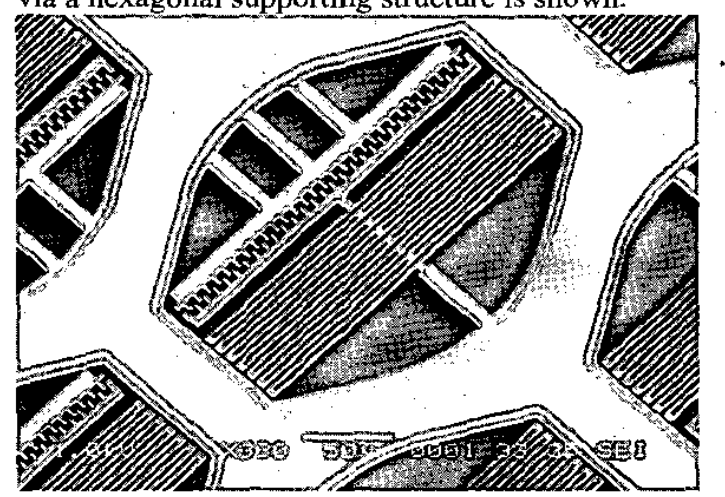

Figure 5: SEM micrograph of a microactuator attached to a hexagonal support structure on a silicon nitride membrane

\section{Operation of the actuators}

Operation with DC voltages resulted in pull-down of the actuators to the membrane. We believe this is caused by charge-trapping in the SiN layer. Therefore individual actuator arrays were actuated with 20 Volt peak to peak AC-signals with varying frequencies. The first experiments were done under atmospheric pressure. The actuators moved over distances of about 1 $\mu \mathrm{m}$; the observed resonance frequency under these circumstances was around $24-26 \mathrm{kHz}$.

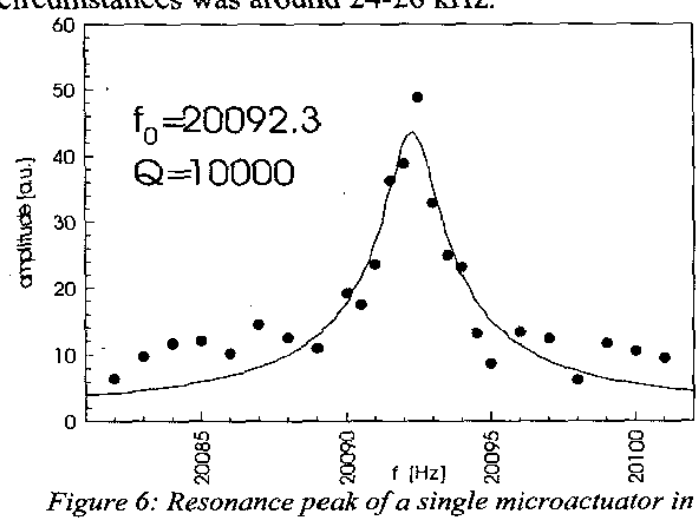

$U H V$

In a later experiment conducted in Cambridge the actuators were placed in vacuum. The pressure during the measurements was $3 \times 10^{-8}$ mbar. The movement of the actuators was measured by a diode laser beam with a spot size of about $30 \mu \mathrm{m}$, which was focused on parts of the comb-drive and the spring. The reflected light was measured with a photo diode (a set-up comparable to the optical read-out of cantilever beams in AFM). The oscillating voltage was produced with a Nanosurf FM Sensor Controller and AFM detector. This electronics is originally made for AC AFM, but here it is used as a very accurate frequency generator (the resolution is about $10 \mathrm{mHz}$ ). The amplitude was $10 \mathrm{mV}$ AC plus $85 \mathrm{mV}$ dc background (hence mechanical motion is now primarily on the same frequency as the 
electrical driving frequency). Results of the measurements are shown in figure 6. A quality factor as high as 10000 was measured. The observed resonance frequency was in the order of $20 \mathrm{kHz}$. The discrepancy between the results of actuation in air and in vacuum could be due to the fact that static electricity that accumulates on the SiN membrane is not removed as quickly in vacuum as in atmospheric conditions. This static electricity introduces an extra force on the actuator which slows it down.

Etching holes in the SiN layer using FIB

After the operation of the actuators was verified the holes in the SiN membrane could be made. This was done using a Focused Ion Beam etcher (FIB). Before the holes could be FIBbed the actuator was covered with a thin layer of carbon by glow sputtering. This is necessary because the FIB can handle only conducting samples. Four large holes of $5 \times 5 \mathrm{~mm}$ were made in the vicinity of the actuators. Their main function is as recognizable markers to find the material that is evaporated through the smaller holes. To show the movement of the spring a series of holes with dimensions of $0.5 \times 0.5 \mathrm{~mm}$ was made just in front of the spring 'beams', close to the center, at the ends of the spring 'arms', and in between these two locations. Between the spring and the comb two other structures were etched: a long slit and a series of holes. The middle of this pattern will always be open, even if the actuator moves over its full length. If the actuator moves, parts of this structure will be closed during a certain percentage of the time the actuator is operating, resulting in a decreased height of the evaporated dot. If the actuator doesn't move the evaporated dot will have the same height everywhere. For this experiment the mobility of the evaporated material on the sample surface will have to be considered; a material that diffuses far over the surface will yield inconclusive results. After FIB the carbon was removed by etching for $10 \mathrm{~min}$ in an oxygen plasma etcher. Although the yield was low (less than $10 \%$ ), some actuators could be moved afterwards.

\section{CONCLUSIONS}

A fabrication process to realize functional structures above thin $\mathrm{SiN}$ membranes has been proposed and successfully demonstrated. Poly-silicon structures were fabricated by sacrificial layer etching on top of a $\mathrm{SiN}$ membrane. Stress in the final structures was minimized by complete removal of the sacrificial $\mathrm{SiO}_{2}$ and by anchoring the poly-silicon structures directly to the $\mathrm{SiN}$ membranes. Using local doping the poly-silicon layers were used for mechanical strengthening, electrical conduction and micro actuation at the same time. As an example of the potential of the technology patterns of hexagonal structures with embedded electrostatic combdrive structures were designed, fabricated and tested. Future work will comprise of the demonstration of the functionality of this enhanced nanostencil technology by deposition experiments.

\section{ACKNOWLEGDGEMENTS}

We would like to thank Meint de Boer for his assistance with polysilicon etching, Frans Segerink for his help with the FIB, and Matthijn de Rooij for his support with white light interferometer measurements. This work was supported by the European Union in the fifth framework programme project "ATOMS" (IST-199914912).

\section{LITERATURE}

[1] J. Brügger, J.W. Berenschot, S. Kuiper, W. Nijdam, B. Otter and M. Elwenspoek, Microelectronic Engineering, 53 (2000), 403-405

[2] J.M van Rijn, G.J. Veldhuis, S. Kuiper; Nanotechnology 9 (1998) 343-345

[3] M. Kölbel, R. W. Tjerkstra, J. Brugger, C. J. M. van Rijn, W. Nijdam, J. Huskens, and D. N. Reinhoudt, Nano Lett., 2 (12) (2002), 1339-1343

[4] M. Kölbel, R.W. Tjerkstra, G. Kim, J. Brugger, C.J.M. van Rijn, W. Nijdam, J. Huskens, and D.N. Reinhoudt, pending for Adv. Funct. Mater.

[5] R. Legtenberg, A.W. Groeneveld and M.C. Elwenspoek, J. Micromech. Microeng., 6 (1996), 320-329.

[6] N. Tas, T. Sonnenberg, H. Jansen, R. Legtenberg, and M. Elwenspoek, J. Micromech. Microeng., Vol. 6, 1996, 385-397.

[7] J.G.E. Gardeniers, H.A.C. Tilmans and C.G.C.J.Visser, Vac. Sci. Technol. A 14, 1996 2879-2892 